\title{
Management of pulmonary aspiration due to undiagnosed achalasia during induction of general anesthesia - A case report -
}

Received October 9, 2021

Revised December 20, 2021

Accepted December 24, 2021

\section{Corresponding author}

Bora Lee, M.D., Ph.D.

Department of Anesthesiology and Pain Medicine, Severance Hospital and Anesthesia and Pain Research Institute, Yonsei University College of Medicine, 50-1 Yonsei-ro, Seodaemun-gu, Seoul 03722, Korea Tel: 82-2-2224-3919

Fax: 82-2-2227-7897

E-mail: dreamkaist@yuhs.ac

\section{Hee Jung Kim, Yong Seon Choi, Jeong Hyun Jin, and Bora Lee}

Department of Anesthesiology and Pain Medicine, Severance Hospital and Anesthesia and Pain Research Institute, Yonsei University College of Medicine, Seoul, Korea

Background: Intraoperative pulmonary aspiration is a rare but potentially fatal complication associated with various risk factors. Preoperative recognition of these risk factors can prevent aspiration events during general anesthesia or facilitate prompt corrective measures in patients experiencing this complication.

Case: A 70-year-old female patient with hypertension underwent bilateral total knee arthroplasty under general anesthesia. Despite using a midnight nothing-per-oral (NPO) protocol, an unpredictable intraoperative aspiration event occurred during anesthesia induction. A detailed evaluation of the patient's medical history and subsequent diagnostic imaging examinations indicated achalasia. She was treated for aspiration pneumonia for 2 weeks. After 2 months, rescheduled total knee arthroplasty was performed under spinal anesthesia without any complications.

Conclusions: Obtaining the patient's medical history and assessing the risk factors are important to prevent unpredictable intraoperative pulmonary aspiration. High-risk patients should undergo adequate preoperative fasting and regional anesthesia or rapid-sequence intubation should be considered for safe induction of general anesthesia.

Keywords: Esophageal achalasia; Esophageal diseases; Pneumonia, aspiration; Respiratory aspiration.
Intraoperative pulmonary aspirations are rare events that occur in approximately one in 2,000 to 3,000 operations, but are potentially fatal complications associated with significant morbidity. Numerous risk factors can cause anesthesia-related aspiration events, including delayed gastric emptying, an incompetent lower esophageal sphincter (LES), need for emergency surgery, and obesity [1]. Among them, achalasia, which is characterized by impaired transit of swallowed food due to LES relaxation disorder and loss of esophageal peristalsis, is a rare disease [2]. The authors experi- enced the occurrence of unpredictable intraoperative aspiration events during anesthesia induction in the patient with no special underlying disease, and the patient was subsequently diagnosed with achalasia after obtaining a detailed medical history and using diagnostic tools. Therefore, we report the management of pulmonary aspiration during induction of anesthesia in one patient and discuss possible measures to prevent intraoperative aspiration.

This is an Open Access article distributed under the terms of the Creative Commons Attribution Non-Commercial License (http://creativecommons.org/licenses/by-nc/4.0) which permits unrestricted non-commercial use, distribution, and reproduction in any medium, provided the original work is properly cited.

Copyright (C) the Korean Society of Anesthesiologists, 2022 


\section{CASE REPORT}

The Institutional Review Board of our hospital waived the need to obtain the patient's informed consent for publication of this case report (no. 4-2021-1071). A 70-year-old female patient (height, $144 \mathrm{~cm}$; weight, $65 \mathrm{~kg}$ ) was admitted to the hospital to undergo bilateral total knee arthroplasty (TKA) for the treatment of degenerative knee joint osteoarthritis. The patient had been previously diagnosed with hypertension, and her preoperative test results were within normal range.

The patient underwent an overnight fast starting at midnight one night before surgery, and entered the operating room at $3 \mathrm{pm}$. The initial vital signs in the operating room were stable: blood pressure, 147/81 mmHg; heart rate, 89 beats/min; and oxygen saturation, $100 \%$. A spinal needle was inserted for spinal anesthesia in the lateral position, but the patient complained of pain and anxiety during the procedure. Therefore, the plan was changed to perform general anesthesia.

Propofol $110 \mathrm{mg}$ was administered intravenously to induce consciousness loss, and $50 \mathrm{mg}$ of rocuronium was injected to relax the muscles. When manual ventilation using a bag and mask began with a peak airway pressure $<10 \mathrm{~cm}$ $\mathrm{H}_{2} \mathrm{O}$, the patient regurgitated food material from the mouth. Therefore, we put the patient in the Trendelenburg position and performed oral suctioning. However, the patient's oxygen saturation decreased to $82 \%$. Additional administration of $30 \mathrm{mg}$ of rocuronium and rapid-sequence intubation were performed with a 6.5-mm tube using a video-guided laryngoscope. After intubation, oxygen saturation was maintained at $95-98 \%$ and arterial line cannulation was performed for continuous vital sign monitoring and arterial blood gas analysis (ABGA), while a substantial amount of food material in the bronchi was suctioned using a bronchoscope. Nasogastric tube placement was performed with a video-guided laryngoscope to identify persistent reflux patterns, and suctioning was also performed (Supplementary Video 1). Additionally, the gastric content and volume were assessed using ultrasonography, but the total gastric volume was lower than $1.5 \times$ the body weight $(\mathrm{kg})$, which is the threshold for an elevated high risk of aspiration. Moreover, the gastric content did not include any solid material (Fig. 1) [3]. The patient was referred for a pulmonology consultation for the treatment of aspiration pneumonia, and a pulmonologist performed fiberoptic bronchoscopy (FOB) in the operating room. Small amounts of foamy secretions were observed in the right lower lobe, and very small amounts of remnant material were aspirated in the bronchi of both lungs through FOB. Lung recruitment maneuvers were performed, and the patient was mechanically ventilated at a tidal volume of 350 $\mathrm{ml}$ and a positive end-expiratory pressure of $5 \mathrm{cmH}_{2} \mathrm{O}$. However, ABGA tests conducted after FOB showed that the $\mathrm{PaO}_{2}$ was $84 \mathrm{mmHg}$ at a $\mathrm{FiO}_{2}$ of 0.6 and peak airway pressure of 15 $\mathrm{cmH}_{2} \mathrm{O}$. Therefore, we decided to cancel the surgery and focus on management of the aspiration pneumonia before rescheduling the surgery. Sugammadex (200 mg) was used to reverse the neuromuscular blockade, and extubation was performed after recovery of spontaneous respiration and consciousness. On interviewing the patient to determine the exact fasting time and obtain a detailed medical history, we found that the patient had experienced severe reflux symptoms, including episodes of waking up after vomiting during sleep, but these were not treated. Low-dose chest computed tomography (CT) examination to determine the cause of vomiting suggested esophageal motility disorder, as well as esophagogastroduodenoscopy (EGD), esophagography, and esophageal manometry were performed to confirm these findings. EGD and esophagography findings showed a dilated esophageal lumen and narrowing of the esophagogastric (EG) junction, and the presence of achalasia were confirmed on manometry, so the patient was diagnosed with achalasia (Fig. 2A, B). Moreover, we suspected aspiration pneumonia in both lungs, especially in the left lower lobe, on the basis of the chest posteroanterior radiographs obtained after anesthesia and treated her with antibiotics for approximately 2 weeks (Fig. 3). She showed no other symptoms other than fever of $37.8^{\circ} \mathrm{C}$ in the general ward, and her oxygen satura-

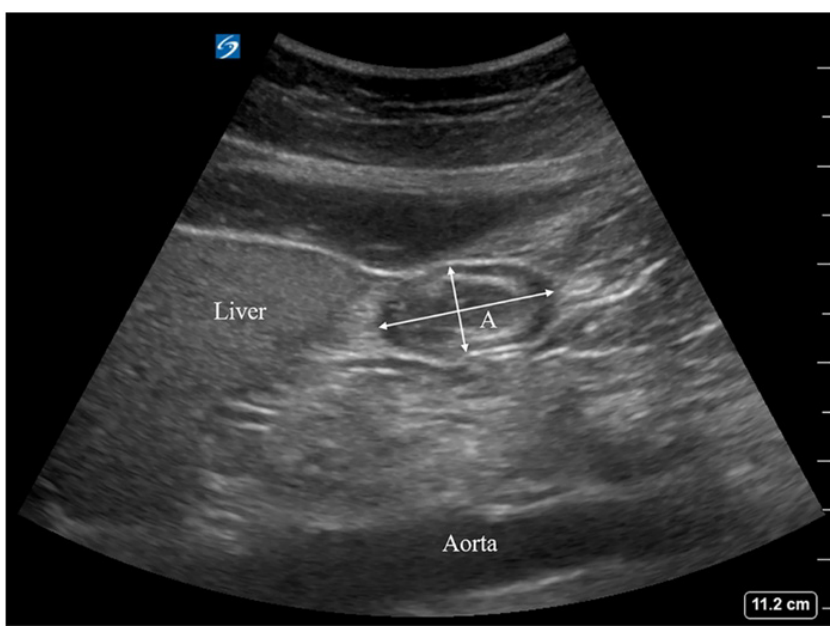

Fig. 1. Ultrasonographic gastric antrum measurement. A: antrum. 

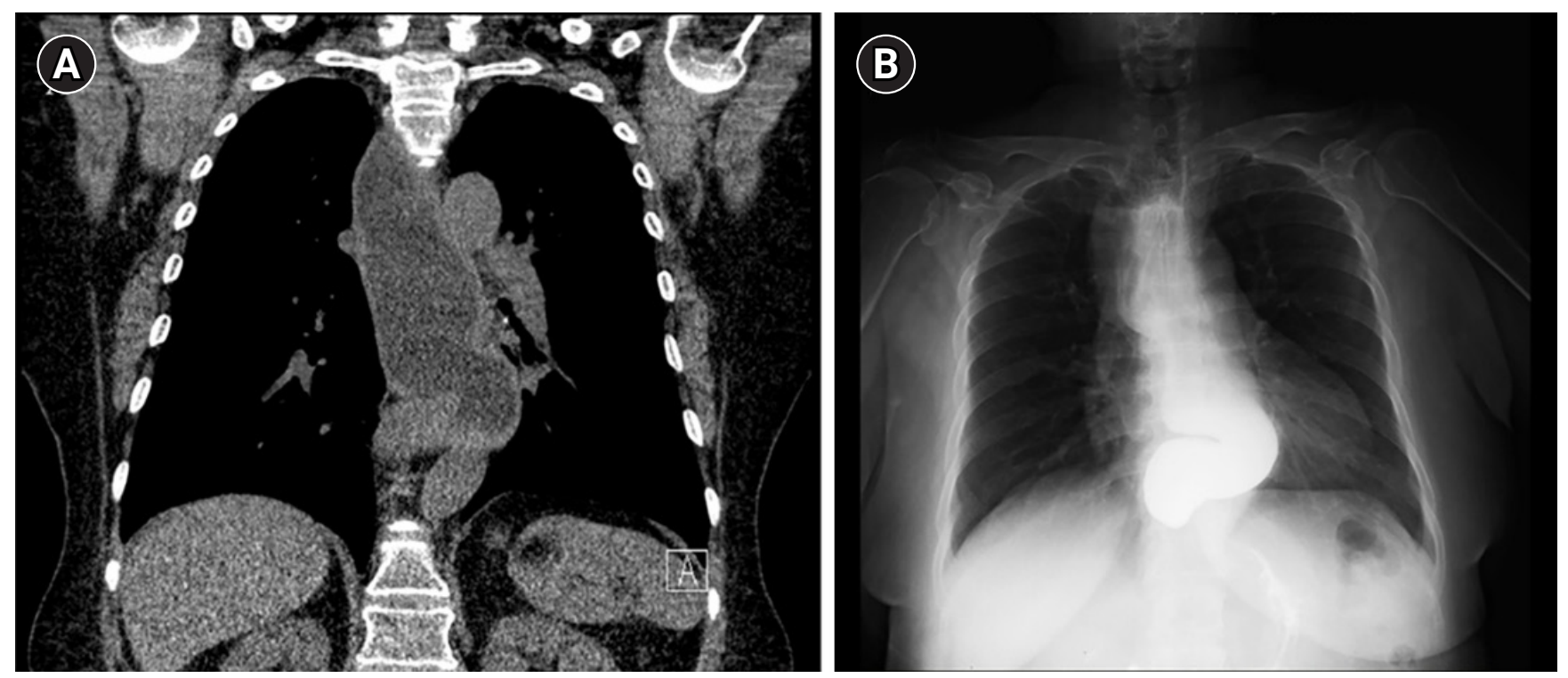

Fig. 2. The diagnostic images. (A) Diffuse and extensive dilatation of the esophagus with narrowing at the esophagogastric junction in chest CT. (B) Marked tortuous dilatation of mid to distal esophagus with abrupt narrowing at the gastroesophageal junction in esophagography (bird-beak sign). CT: computed tomography.
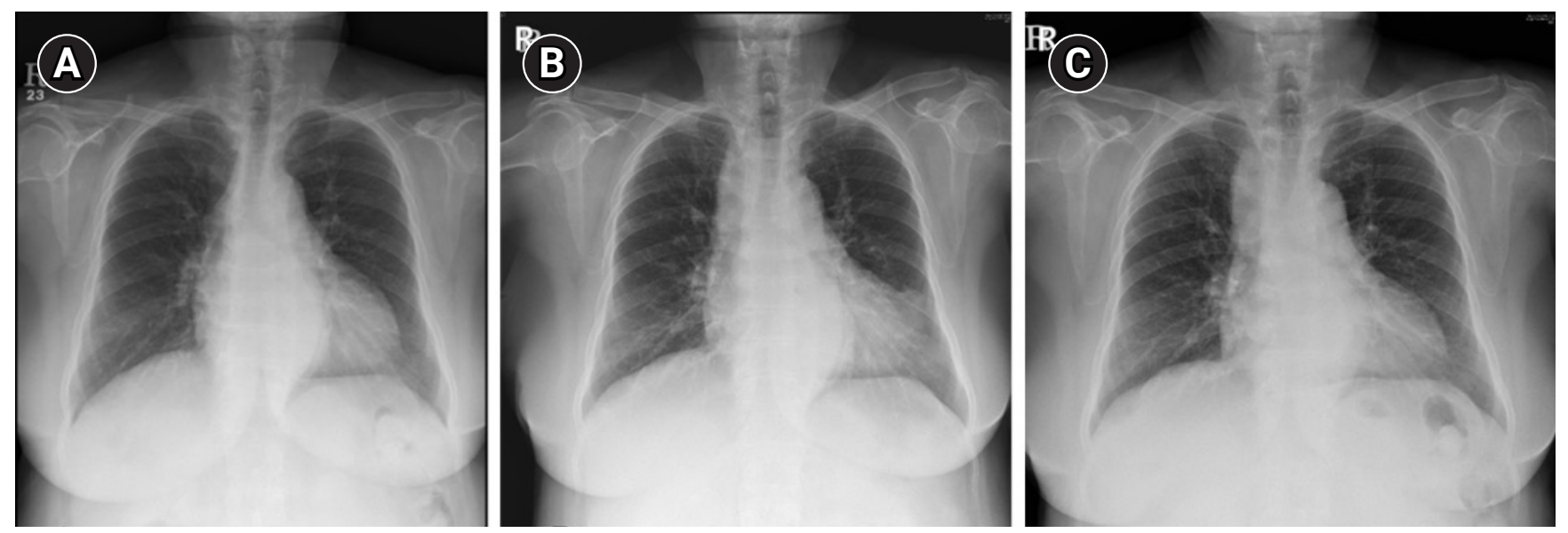

Fig. 3. Posteroanterior chest radiographs. (A) Preoperative image; (B) diffuse consolidation on both lungs on postoperative day 3; (C) improved consolidation at 4 weeks postoperatively.

tion was maintained over $95 \%$ by administering oxygen through a nasal cannula at a rate of $3 \mathrm{~L} / \mathrm{min}$ and tapering off the next day. The patient was discharged after a 6-day hospital stay and prescribed oral antibiotics for 1 week.

She was hospitalized 2 months later for the rescheduled TKA. The patient was put on a soft-food diet from 2 days before the surgery, and EGD was performed at $5 \mathrm{pm}$ on the day before the surgery to remove a large amount of water and food material from the esophagus. No food material remained in the stomach. Ultrasound-guided spinal anesthesia was successfully performed in a sitting position. The patient underwent bilateral TKA without any complications and was discharged on postoperative day 2.

\section{DISCUSSION}

Intraoperative pulmonary aspiration is a rare complication, but it may cause serious sequelae. The predisposing risk factors for anesthesia-related aspiration include a full stomach, delayed gastric emptying, an incompetent LES, esophageal cancer, previous esophageal surgery, gastrointestinal obstruction, need for emergency surgery, lack of coordination of swallowing or respiration, and obesity [1]. Among these, achalasia is a rare disease caused by the in- 
complete relaxation of the LES and the loss of esophageal peristalsis. Its incidence and prevalence in Korea have been reported to be 0.4 and 6.3 per 100,000 individuals, respectively [4]. The clinical presentations of this condition may include dysphagia, regurgitation, chest pain, weight loss, and odynophagia.

In the present case, the patient was diagnosed with achalasia because of regurgitation of food materials during induction of general anesthesia, although she showed no specific underlying disease other than hypertension. A detailed evaluation of her medical history revealed occasional episodes of severe reflux symptoms, including vomiting during sleep. Using the Eckardt Symptom score [5], the patient's symptoms were assigned to the subsets of no dysphagia and occasional regurgitation, no retrosternal pain, and weight loss of $<5 \mathrm{~kg}$, each of which was rated at 2 points, with scores $\geq 3$ considered suggestive of active achalasia [6]. According to the 2019 Seoul consensus on esophageal achalasia guidelines [5], endoscopy and CT/endoscopic ultrasound (EUS) can be performed to diagnose patients with suspected achalasia. In this patient, low-dose chest CT indicated esophageal motility disorder, while EGD showed a dilated esophageal lumen and a tightly closed EG junction, which indicated achalasia. Furthermore, esophagography and high-resolution manometry were recommended to confirm achalasia. The esophagographic findings were dilation of the esophagus, narrowing at the EG junction with a bird-beak appearance, and incomplete LES relaxation. After esophageal manometry, this patient was diagnosed with Chicago classification achalasia subtype 2 [5]. This subtype is the most common type, and is characterized by the absence of peristalsis along with an abnormal pan-esophageal pressurization and a good response to pneumatic dilation. Considering the patient's advanced age and the severity of the disease, pneumatic balloon dilation was recommended as an initial treatment before the knee surgery. However, the patient wished to undergo orthopedic surgery first. Therefore, follow-up treatment of achalasia was planned after the surgery.

Prevention is the ideal approach for the management of intraoperative pulmonary aspiration. Thus, identification of predisposing factors and risk assessment through a thorough preoperative physical examination and a review of the current symptoms are important [1]. The predisposing risk factors can be classified as patient-related, operative, and anesthetic factors. Patient-related factors include a full stomach, delayed gastric emptying, an incompetent LES, and esophageal diseases; operative factors include upper gastrointestinal surgery and laparoscopy; and anesthetic factors include light anesthesia, supraglottic airways, positive-pressure ventilation, and a difficult airway. Preoperative fasting for a few days before the procedure is recommended in cases where the patient is expected to show poor gastric emptying and retention of solid food matter in the stomach [1]. For patients at a high risk of achalasia undergoing peroral endoscopic myotomy (POEM) procedures, preoperative EGD may reduce the aspiration risk, while preemptive nasogastric tube placement may also be an alternative approach [7].

If aspirations occur despite proper application of preventive measures, rapid-sequence induction and management of the cricoid pressure for prompt placement of an endotracheal tube to secure the airway are critical [8]. The cricoid pressure should be reduced if this practice interferes with airway management [9]. After securing the airway, FOB should be considered for endotracheal suctioning. Subsequently, a decision regarding continuation of the surgery can be taken on the basis of the patient's oxygen saturation, pulmonary compliance, and response to bronchodilator and positive end-expiratory pressure. Although antibiotics and steroids are not used routinely, they can be used if necessary.

Anesthesia management in patients diagnosed with achalasia has been covered in some previous case reports. Creagh-Barry et al. [10] reported performing intubation with cricoid pressure along with fasting for solids and fluid for 12 $\mathrm{h}$ before the operation, $45^{\circ}$ reverse Trendelenburg positioning during induction of anesthesia, and extubation with clear consciousness without any complications. Their findings showed that reducing the contents of the esophagus through nasogastric tube insertion can lower the risk of pulmonary aspiration. However, Choi et al. [11] reported a case of regurgitation and aspiration pneumonitis even after preoperative fasting for $12 \mathrm{~h}$ in a patient who had undergone a POEM procedure 6 months before surgery. In our case, despite $16 \mathrm{~h}$ of fasting, the aspiration events occurred, and immediate treatments such as Trendelenburg positioning, oral suction, rapid-sequence intubation, endotracheal suctioning through a bronchoscope, nasogastric tube insertion, and FOB in the operating room were performed. Takenaka et al. [12] reported that a $10^{\circ}$ Trendelenburg position with the Sellick position or more than $35^{\circ}$ Trendelenburg positioning in simple neck extension had the potential to completely prevent aspiration. However, this position cannot be used in patients with cervical spine instability and may make trache- 
al intubation difficult. When general anesthesia is provided to patients at high risk of pulmonary aspiration, it is important to prevent pulmonary aspiration and adopt an optimal posture for rapid-sequence intubation [13]. When the patients were hospitalized for reoperation, EGD was performed on the day before the surgery to remove a large amount of water and food materials from the esophagus. Subsequently, ultrasound-guided spinal anesthesia was performed successfully, and the patient underwent bilateral TKA without facing any complications.

Another notable point in the present case is related to the usefulness of imaging techniques. Recently, gastric ultrasound was reported to be useful to identify or rule out a full stomach and assess the risk of pulmonary aspiration [14,15]. However, gastric ultrasound cannot predict a high risk of pulmonary aspiration in patients with esophageal mobility disorder, similar to our patient. In the EGD performed following anesthesia, a substantial amount of food material was found only in the esophagus, not in the stomach. In patients with undiagnosed esophageal diseases, the risk of pulmonary aspiration can only be predicted by detailed interviews. The patient in this case was not diagnosed with achalasia, but experienced severe reflux symptoms such as vomiting during sleep, indicating that a detailed evaluation of the patient's medical history before anesthesia is important.

Symptoms related to a high risk of pulmonary aspiration include gastrointestinal reflux disease, esophageal dysmotility, difficulty in swallowing, gas bloating, and other signs of delayed gastric emptying [1]. Further, patients that are considered to be at a high risk of pulmonary aspiration should receive an adequately detailed explanation of aspiration and undergo appropriately long preoperative fasting. The current guidelines recommend consumption of a light meal and clear liquid up to 6 and $2 \mathrm{~h}$, respectively, prior to the surgery [16]. However, there is limited knowledge concerning the appropriate fasting time in surgical patients at a high risk of anesthesia-related regurgitation and aspiration. Although it is safe to maintain consciousness through regional anesthesia, in cases requiring general anesthesia, preoperative EGD or nasogastric tube insertion is necessary, and rapid-sequence intubation should be considered to allow safe induction of anesthesia.

If the patient is judged to be at high risk, adequate preoperative fasting and regional anesthesia or rapid-sequence intubation should be considered for the safe induction of general anesthesia.

\section{SUPPLEMENTARY MATERIALS}

Supplementary video is available at https://doi.org/ 10.17085/apm.21102.

\section{FUNDING}

None.

\section{CONFLICTS OF INTEREST}

Yong Seon Choi has been an editor of the Anesthesia and Pain Medicine since 2018; however, she was not involved in the peer reviewer selection, evaluation, or decision process of this article. No other potential conflicts of interest relevant to this article were reported.

\section{DATA AVAILABILITY STATEMENT}

None.

\section{AUTHOR CONTRIBUTIONS}

Conceptualization: Yong Seon Choi, Bora Lee. Data curation: Hee Jung Kim, Jeong Hyun Jin. Formal analysis: Hee Jung Kim. Methodology: Yong Seon Choi, Bora Lee. Writing - original draft: Hee Jung Kim. Writing - review \& editing: Hee Jung Kim, Yong Seon Choi, Bora Lee. Investigation: Jeong Hyun Jin. Resources: Jeong Hyun Jin. Supervision: Bora Lee. Validation: Yong Seon Choi.

\section{ORCID}

Hee Jung Kim, https://orcid.org/0000-0002-2143-3943 Yong Seon Choi, https://orcid.org/0000-0002-5348-864X Jeong Hyun Jin, https://orcid.org/0000-0002-5348-864X Bora Lee, https://orcid.org/0000-0002-7699-967X

\section{REFERENCES}

1. Nason KS. Acute intraoperative pulmonary aspiration. Thorac Surg Clin 2015; 25: 301-7.

2. Vaezi MF, Pandolfino JE, Yadlapati RH, Greer KB, Kavitt RT. ACG clinical guidelines: diagnosis and management of achalasia. Am J Gastroenterol 2020; 115: 1393-411.

3. Van de Putte P, Perlas A. Ultrasound assessment of gastric content and volume. Br J Anaesth 2014; 113: 12-22.

4. Kim E, Lee H, Jung HK, Lee KJ. Achalasia in Korea: an epidemi- 
ologic study using a national healthcare database. J Korean Med Sci 2014; 29: 576-80.

5. Jung HK, Hong SJ, Lee OY, Pandolfino J, Park H, Miwa H, et al. Korean Society of Neurogastroenterology and Motility. 2019 Seoul consensus on esophageal achalasia guidelines. J Neurogastroenterol Motil 2020; 26: 180-203.

6. Taft TH, Carlson DA, Triggs J, Craft J, Starkey K, Yadlapati R, et al. Evaluating the reliability and construct validity of the Eckardt symptom score as a measure of achalasia severity. Neurogastroenterol Motil 2018; 30: e13287.

7. Löser B, Recio Ariza O, Saugel B, Reuter DA, Zöllner C, Werner $\mathrm{YB}$, et al. Anesthesia for patients undergoing peroral endoscopic myotomy procedures: a review of the literature. Anesth Analg 2020; 130: 1331-40.

8. Kluger MT, Visvanathan T, Myburgh JA, Westhorpe RN. Crisis management during anaesthesia: regurgitation, vomiting, and aspiration. Qual Saf Health Care 2005; 14: e4.

9. Butler J, Sen A. Towards evidence-based emergency medicine: best BETs from the Manchester Royal Infirmary. BET 1: cricoid pressure in emergency rapid sequence induction. Emerg Med J 2013; 30: 163-5.

10. Creagh-Barry P, Parsons J, Pattison CW. Achalasia and anaes- thesia, a case report. Anaesth Intensive Care 1988; 16: 371-3.

11. Choi JW, Lee JH, Kim EJ, Lee SG, Ban JS, Min BW. Aspiration pneumonitis in achalasia patient treated by per oral endoscopic myotomy: a case report. Anesth Pain Med 2012; 7: 151-4.

12. Takenaka I, Aoyama K, Iwagaki T. Combining head-neck position and head-down tilt to prevent pulmonary aspiration of gastric contents during induction of anaesthesia: a volunteer and manikin study. Eur J Anaesthesiol 2012; 29: 380-5.

13. Higgs A, McGrath BA, Goddard C, Rangasami J, Suntharalingam G, Gale R, et al. Difficult Airway Society; Intensive Care Society; Faculty of Intensive Care Medicine; Royal College of Anaesthetists. Guidelines for the management of tracheal intubation in critically ill adults. Br J Anaesth 2018; 120: 323-52.

14. Kruisselbrink R, Gharapetian A, Chaparro LE, Ami N, Richler D, Chan VWS, et al. Diagnostic accuracy of point-of-care gastric ultrasound. Anesth Analg 2019; 128: 89-95.

15. Haskins SC, Kruisselbrink R, Boublik J, Wu CL, Perlas A. Gastric ultrasound for the regional anesthesiologist and pain specialist. Reg Anesth Pain Med 2018; 43: 689-98.

16. Fawcett WJ, Thomas M. Pre-operative fasting in adults and children: clinical practice and guidelines. Anaesthesia 2019; 74: 83-8. 